\title{
Neuroprotective effect of three caffeic acid derivatives via ameliorate oxidative stress and enhance PKA/CREB signaling pathway
}

\author{
Wei Fu ${ }^{\mathrm{a}}$, Hongyan Wang ${ }^{\mathrm{b}}$, Xiuhua Ren ${ }^{\mathrm{a}}$, Hengyi $\mathrm{Yu}^{\mathrm{a}}$, Yongfang Lei ${ }^{\mathrm{a}}$, Qian Chen ${ }^{\mathrm{a}, *}$ \\ a Department of Pharmacy, Tongji Hospital Affiliated to Tongii Medical College, Huazhong University of Science and Technology, Wuhan 430030, Hubei Province, PR \\ China \\ ${ }^{\mathrm{b}}$ Department of Periodontics, School of Stomatology, China Medical University, Shenyang 110002, Liaoning Province, PR China
}

\section{A R T I C L E I N F O}

\section{Keywords:}

Caffeic acid derivatives

D-Galactose (D-gal)

Antioxidant

PKA/CREB signaling pathway

Neurodegenerative diseases

\begin{abstract}
A B S T R A C T
This study was conducted to elucidate the neuroprotective effect of caffeic acid phenethyl ester (CAPE), (R)-2Hydroxy-3-(3,4-dihydroxyphenyl) propionic acid (Danshensu) and Curcumin, three caffeic acid derivatives which are contained in fruits, grains and certain dietary supplements.

Our results showed that these compounds significantly attenuated $\mathrm{H}_{2} \mathrm{O}_{2}$-induced toxicity in PC12 cells in a dose-dependent manner. Furthermore, these compounds significantly improved the behavioral performance of Dgal-treated mice in both step-down avoidance test and Morris water maze test. Biochemical examination and western blot analysis showed that these compounds could ameliorate oxidative stress and facilitate activation of the protein kinase A (PKA)-cyclic AMP response element-binding protein (CREB) pathway.

Its beneficial effects may partly relate to enhancing the activity of endogenous antioxidant enzymes and modulating the PKA/CREB signaling pathway. Furthermore, our results also indicated that the presence of 3, 4dihydroxyphenyl groups in A ring may enhance their neuroprotective activity.
\end{abstract}

\section{Introduction}

It has been proposed that formation of reactive oxygen species (ROS) plays an important role in neuronal death in a variety of agerelated neurodegenerative disorders such as Alzheimer's disease and Parkinson's disease $[1,2]$. In a physiological condition, ROS are formed as a natural byproduct of the normal metabolism of oxygen and have important roles in cell signaling and homeostasis. However, in term of environmental stress, increased ROS production coupled with a reduced antioxidant defense has been postulated to play a critical role in brain injury [3]. ROS can cause enormous damage to the structure and the function of the cell membranes and even lead to the oxidative damage of the tissues and organs. It has been shown that the hippocampus was one of the most vulnerable area in the brain to oxidative stress, and the continuously damaged neuronal cells in hippocampus will eventually trigger neurodegenerative disorders [4].

Furthermore, recent research indicated that the increase in ROS modulates a number of signaling targets in the nervous system, including the protein kinase A(PKA) and cyclic AMP response element binding protein (CREB) [5]. The PKA/CREB signaling pathway has been reported to play a critical role in learning and memory [6]. PKA can phosphorylate CREB at serine 133, which can lead to trans-synaptic signaling and regulates the expression of genes that are important for synaptic plasticity mediating long-term memory formation. It is reported that CREB-mediated gene expression is impaired in the brain of both $\mathrm{AD}$ mouse models and patients $[7,8]$.

Caffeic acid, one of the most common phenolic acids, is widely distributed in several species of plants and regularly consumed in human diet $[9,10]$. Caffeic acid and derivatives have been extensively studied in the past years, with mounting evidence showing that these chemicals have a variety of functions including acting as antioxidant, suppressing cerebral lipid peroxidation, and reducing brain infarct after cerebral ischemia in rats $[11,12]$. It has been also suggested that caffeic acid and its derivatives have therapeutic potential in the treatment of neurodegenerative diseases [13].

Three caffeic acid derivatives named CAPE (Caffeic acid phenethyl ester), Danshensu ((R)-2-Hydroxy-3-(3,4-dihydroxyphenyl) propionic acid), and Curcumin are frequently found in fruits, human grains and dietary supplements for human consumption, and also found in traditional Chinese medicinal herbs [14]. The chemical structures of CAPE, Danshensu and Curcumin are shown in Fig. 1. One of the most prominent structural similarities among these three molecules is that

\footnotetext{
*Corresponding author at: Department of pharmacy, Tongji Hospital Affiliated to Tongji Medical College, Huazhong University of Science and Technology, 1095 Jiefang Avenue, Wuhan 430030, Hubei Province, PR China.

E-mail address: lui91982@gmail.com (Q. Chen).
} 
<smiles>O=C(/C=C/c1ccc(O)c(O)c1)OCCc1ccccc1</smiles>

CAPE<smiles>O=C(O)C(O)Cc1ccc(O)c(O)c1</smiles>

Danshensu

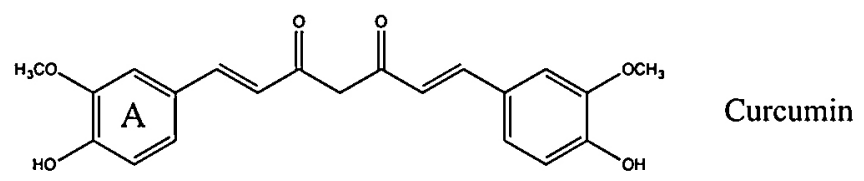

Fig. 1. Structures of the caffeic acid derivatives CAPE, Curcumin and Danshensu.

they all contain an aromatic ring (A). They differ, as shown in Fig. 1, exists in the numbers of hydroxyl group in A ring and the conjugated double bond.

A large number of studies have been carried out on CAPE, Danshensu and Curcumin individually, but no work has been conducted to compare the neuroprotective effects of the three above mentioned caffeic acid derivatives or the possible structure-bioactivity relationship. Therefore, the aim of this study was to investigate the protective effect of these three caffeic acid derivatives against $\mathrm{H}_{2} \mathrm{O}_{2}$-induced oxidative damage in PC12 cells and D-galactose (D-gal)-induced cognitive impairment in mice. This study also made a preliminary exploration of whether the hydroxyl groups in the A ring or conjugated double bond influence their neuroprotective effect.

\section{Materials and methods}

\subsection{Reagents}

CAPE, Danshensu and Curcumin (purity $>98 \%$ ) were purchased from Nanjing Mangesi Biotechnological Co., Ltd., (Nanjing, Jiangsu province, PR China). D-gal was purchased from Sigma-Aldrich (St. Louis, MO, USA) and dissolved in physiological saline. Commercial kits used for the determination of malondialdehyde (MDA), superoxide dismutases (SOD), glutathione peroxidase (GPx) and catalase were purchased from Jiancheng Institute of Biotechnology (Nanjing, Jiangsu province, PR China). Antibodies against total PKA, phospho-PKA, total CREB and phospho-CREB were purchased from Cell Signaling Technology (SanFrancisco, CA, USA).

\subsection{Cell culture and treatment}

PC12 cells were grown in Dulbecco's Modified Eagle's medium (DMEM) supplemented with $10 \%$ fetal bovine serum, $5 \%$ heat-inactivated horse serum, 100 units $/ \mathrm{mL}$ penicillin, and $100 \mu \mathrm{g} / \mathrm{mL}$ streptomycin at $37{ }^{\circ} \mathrm{C}$ in an incubator aerated with $95 \%$ air and $5 \% \mathrm{CO}_{2}$. All experiments were carried out within 24-48 h after cells were seeded onto plates or dishes. Concentrations of $200 \mu \mathrm{M} \mathrm{H}_{2} \mathrm{O}_{2}$ and vehicle as control, and then three caffeic acid derivatives were dissolved and diluted with serum free medium. To produce oxidative stress, $\mathrm{H}_{2} \mathrm{O}_{2}$ was freshly prepared from $30 \%$ stock solution prior to each experiment.

\subsection{Viability assay}

PC12 cells were plated at about density of $1 \times 10^{4}$ cells/well in 96well plates and measured by MTT assay [15]. Briefly, each compound $(0.1,0.5,1,5$ and $10 \mu \mathrm{M})$ were pre-incubated for $24 \mathrm{~h}$ before $200 \mu \mathrm{M}$
$\mathrm{H}_{2} \mathrm{O}_{2}$ was added. After $4 \mathrm{~h}$ exposure to $\mathrm{H}_{2} \mathrm{O}_{2}$, MTT solution in phosphate-buffered saline (PBS) was added to attain a final concentration of $0.5 \mathrm{mg} / \mathrm{mL}$, and the incubation continued for $4 \mathrm{~h}$. Finally, MTT was removed and the colored formazan was dissolved in dimethyl sulfoxide (DMSO). The absorption values were measured at $570 \mathrm{~nm}$ using a DigiScan Microplate Reader (Assys Hitech Kornenburg, Austria). The viability of PC12 cells in each well was presented as the percentage of control cells.

\subsection{Animals and administration}

The Kunming mice, weighing 18-22 g, were obtained from Experimental Animal Center, Tongji Medical college, Huazhong University of Science and Technology, China. The animals were adapted to the feeding condition for a week, with controlled temperature $\left(22 \pm 2{ }^{\circ} \mathrm{C}\right)$ and humidity $(60 \%)$. They were fed with standard diet and water ad libitum. After acclimatization to the laboratory conditions, the mice were randomly divided into five groups (12 in each group): vehicle control group, D-gal model group, CAPE group, Danshensu group and Curcumin group. The mice of D-gal model group, CAPE group, Danshensu group and Curcumin group were daily subcutaneously injected with D-gal at the dose of $150 \mathrm{mg} / \mathrm{kg}$ once daily for 7 weeks while those of vehicle control group were treated with same volume of physiological saline. From the sixth week, CAPE group, Danshensu group and Curcumin group mice were intraperitoneal (i.p.) injected with CAPE, Danshensu, Curcumin at the dose of $10 \mathrm{mg} / \mathrm{kg}$ respectively after the injection of D-gal for two weeks (the sixth and seventh week). Vehicle control group and D-gal model group mice were administered with same volume of physiological saline. At the end of the treatment period, behavioral tests and biochemical measurements were performed as follows. All experiments were performed in compliance with the Chinese legislation on the use and care of laboratory animals and were approved by the Huazhong University of Science and Technology Committee on Animal Care and Use.

\subsection{Step-down avoidance test}

The apparatus consisted of a plastic box divided into five equal cabins with grid floor made of stainless steel bar spaced $1 \mathrm{~cm}$ apart. The rubber column platform ( $4.5 \mathrm{~cm}$ in diameter and $4.5 \mathrm{~cm}$ in height) was fixed in one corner of the cabins. Initially, a mouse was placed on the safe platform. When it stepped down with all the four paws onto the floor, it would receive a foot shock $(36 \mathrm{~V}, 0.3 \mathrm{~mA}, 2 \mathrm{~s})$ and it was normal reaction to escape back onto the platform. This test was performed after the Morris water maze test as previously described [33]. At the beginning of training, the mice were placed in the box to adapt for $5 \mathrm{~min}$. Next, electric currents were delivered and the mice would jump onto the platform to avoid the electric shock, and the electric currents were maintained for $3 \mathrm{~min}$. After a $24 \mathrm{~h}$ interval, the mice were again placed on the platform, step-down latency (duration of staying on the platform) in $s$ and the number of errors (the mouse steps down from the platform and gets a shock) within 3 min were measured as learning performances.

\subsection{Morris water maze test}

The experimental apparatus consisted of a circular water tank $\left(120 \mathrm{~cm}\right.$ in diameter, $50 \mathrm{~cm}$ in height), containing water $\left(22 \pm 1{ }^{\circ} \mathrm{C}\right)$ to a depth of $30 \mathrm{~cm}$, which was rendered opaque by adding black nontoxic carbon ink. The tank was divided into four equal quadrants, an escape platform was hidden $1 \mathrm{~cm}$ below the surface of the water and placed at the midpoint of one quadrant. During the test trial, the mouse was placed in the water facing the wall at one of three randomized starting positions (in three different quadrants that did not contain the platform). It was given $90 \mathrm{~s}$ to find the platform and was allowed to rest on it for $10 \mathrm{~s}$. The animals failed to find the location within the given 
time were gently guided to the platform and were allowed to stay on it for $10 \mathrm{~s}$. Each mouse was given four trials daily for five consecutive days, with an inter-trial interval of about $60 \mathrm{~s}$. The escape latency to locate the platform, and the swimming distance were recorded. The mean data from daily test were used for statistical analysis.

To determine whether the animal would take a spatial learning strategy to locate the platform, a single spatial probe trial was assessed on day 6 , the platform was removed from the water tank, and the mice were allowed to swim freely for $60 \mathrm{~s}$. The number of crossing the nonexits (the previously platform site) was recorded, and the time that a mouse spent in the target quadrant (where the platform was located) was measured for each trial. All data were recorded and analyzed by a computerized video imaging analysis system (EthoVision, Noldus Information Technology BV, Wageningen, The Netherlands).

\subsection{Biochemical analysis}

For biochemical studies, mice were deeply anesthetized by chloral hydrate and sacrificed by decapitation after behavioral testing. Brains were removed and dissected on ice to obtain the hippocampus. Before detection, the hippocampus was homogenized in cold saline. The homogenate $(10 \%)$ was centrifuged at $4000 \mathrm{~g}$ at $4{ }^{\circ} \mathrm{C}$ for $15 \mathrm{~min}$, and the supernatant was used for assay. Protein concentration was determined via the Coomassie blue protein-binding assay using bovine serum albumin (BSA) as a standard [16].

MDA was measured at the wavelength of $532 \mathrm{~nm}$ by reacting with thiobarbituric acid (TBA) to form a stable chromophoric production [17]. MDA content was expressed as nanomoles per milligram of brain protein. SOD activity was detected based on its ability to inhibit the oxidation of oxymine by the xanthine/xanthine oxidase system [18]. One unit (U) of SOD activity was defined as the amount that reduced the absorbance at $550 \mathrm{~nm}$ by $50 \%$, and data were expressed as units per microgram of brain protein. The activity of GPx was determined by quantifying the rate of oxidation of the reduced glutathione to the oxidized glutathione by $\mathrm{H}_{2} \mathrm{O}_{2}$ catalyzed by GPx [19]. One unit of GPx was defined as the amount of enzyme that reduced the level of GSH by $1 \mu \mathrm{M}$ in $1 \mathrm{~min}$ per milligram protein. Catalase activity was measured by employing hydrogen peroxide to generate $\mathrm{H}_{2} \mathrm{O}$ and $\mathrm{O}_{2}$ [20]. Catalase activity was calculated as $\mathrm{nM} \mathrm{H}_{2} \mathrm{O}_{2}$ consumed in 1 min per milligram protein.

\subsection{Western blot analysis}

Briefly, protein samples were separated by $10 \%$ SDS-polyacrylamide gel electrophoresis and then transferred to a PVDF membrane (Roche Diagnostics Corporation, Indianapolis, IN, USA) by electrophoretic transfer (Bio-Rad Laboratories, Inc., USA). Transferred membranes were blocked for $1 \mathrm{~h}$ at room temperature with $5 \%$ nonfat milk in Tris-buffered saline containing $0.1 \%$ Tween 20 (TBST), and then incubated overnight at $4{ }^{\circ} \mathrm{C}$ with different primary antibodies. After three washes with TBST, the membranes were incubated with horseradish peroxidase-conjugated secondary antibodies in TBST with $3 \%$ nonfat milk for $1 \mathrm{~h}$ at room temperature. Immunoblots were developed on films using the enhanced chemiluminescence technique (Super Signal West Pico; Pierce Biotechnology, Rockford, IL, USA). Quantification of bands was determined by densitometric analysis using Bio-Rad Quantity One. The data were normalized using GAPDH as an internal control and standardized with the vehicle control as 1.0.

\subsection{Statistical analysis}

Results were presented as mean \pm S.E.M. Group differences in the escape latency and the swimming distance in the Morris water maze training task were analyzed with two-way analysis of variance (ANOVA) with repeated measures, the factors being treatment and training day. The other data were analyzed using one-way ANOVA

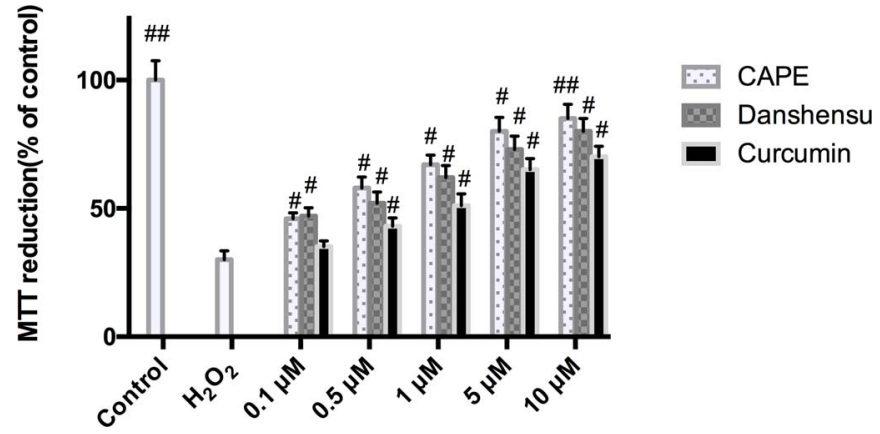

Fig. 2. Protective effect of three caffeic acid derivatives against $\mathrm{H}_{2} \mathrm{O}_{2}$-induced PC12 cell injury. The data are presented as means \pm S.E.M. $(\mathrm{n}=6) .{ }^{\#} p<0.05$ and ${ }^{\# \#} p<0.01$ compared with $\mathrm{H}_{2} \mathrm{O}_{2}$ group.

followed by the Student-Newman-Keuls test. A criterion of $p<0.05$ was accepted as statistically significant.

\section{Results}

\subsection{Effect of three caffeic acid derivative against $\mathrm{H}_{2} \mathrm{O}_{2}$-induced cytotoxicity in PC12 cells}

$\mathrm{H}_{2} \mathrm{O}_{2}$-induced PC12 cells injury was assessed by measuring reduced MTT activity. Cell viability was reduced when treated with $\mathrm{H}_{2} \mathrm{O}_{2}$. When PC12 cells were treated with $200 \mu \mathrm{M}$ of $\mathrm{H}_{2} \mathrm{O}_{2}$ for $4 \mathrm{~h}$, their survival rate was about $30.4 \pm 4.1 \%$ (Fig. 2). Treatment with 0.1, 0.5, 1, 5 and $10 \mu \mathrm{M}$ concentration of CAPE increased the viability of PC12 cells significantly to $46.4 \pm 4.6,57.7 \pm 6.3,67.1 \pm 5.7,80.2 \pm 9.3$ and $85.1 \pm 8.9 \%$, respectively $(p<0.05$ or $p<0.01)$. Similarly, treatment with $0.1,0.5,1,5$ and $10 \mu \mathrm{M}$ concentration of Danshensu increased the viability of PC12 cells significantly to $47.3 \pm 5.2$, $52.7 \pm 7.1,61.8 \pm 5.9,73.2 \pm 6.3$ and $80.6 \pm 7.1 \%$, respectively $(p<0.05$ or $p<0.01)$. Treating PC12 cells with $0.5,1,5$ and $10 \mu \mathrm{M}$ concentration of Curcumin also increased the viability of PC12 cells significantly to $43.4 \pm 3.9,51.2 \pm 5.3,65.2 \pm 4.7$ and $70.2 \pm 6.3$, respectively $(p<0.05$ or $p<0.01$ ), but $0.1 \mu \mathrm{M}$ concentration of Curcumin did not show any significant effect in improving the survival of PC12 cells.

\subsection{Step down test}

We used step-down avoidance test to evaluate the effect of these three caffeic acid derivatives on memory formation in mice. As shown in Fig. 3, the D-gal model mice exhibited reduced latency $(p<0.01)$ and increased number of errors $(p<0.01)$ compared to the vehicle control mice. All the three caffeic acid derivatives could significantly prolong the latency and decrease the number of errors as compared with the D-gal model $(p<0.05$ or $p<0.01)$. Furthermore, it is obvious that CAPE exhibited highest effect and Curcumin exhibited lowest effect among the three caffeic acid derivatives.

\subsection{Morris water maze test}

Morris water maze test was used to assess the effects of these three caffeic acid derivatives on impairment of spatial learning and memory. The performance of all treated groups was improved with training. As shown in Fig. 4A, there were significant differences in mean latency of the animal between training days $[\mathrm{F}(4,205)=29.346, p<0.001]$ and between treatments $[\mathrm{F}(4,205)=45.843, p<0.001]$, but no interaction between the factors day and treatment $[F(16,205)$ $=0.535, p>0.05]$. The $\mathrm{D}$-gal model group took longer to find the hidden platform in all training days as compared with the vehicle control group ( $p<0.001$ ), suggesting that the D-gal model mice had significant cognitive impairment. Meanwhile, Fig. 4B shows the swim- 
(A)

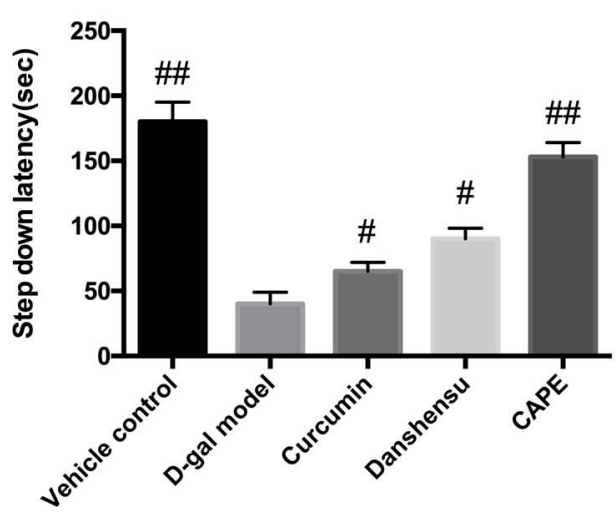

(B)

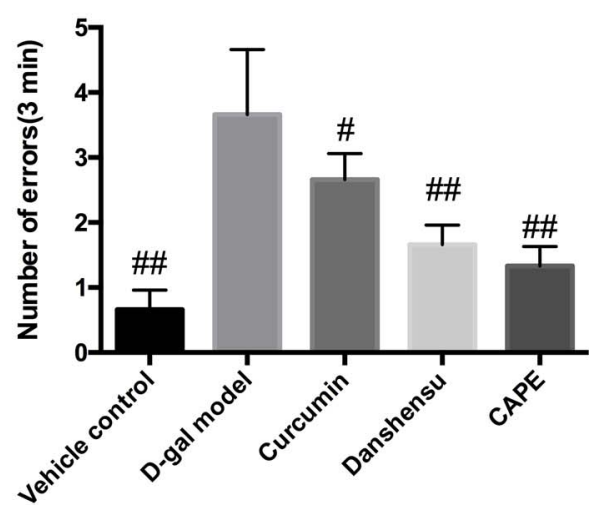

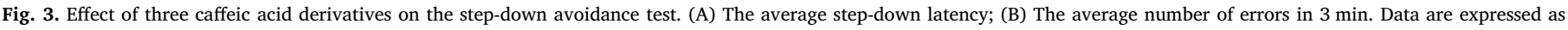
means \pm SEM $(\mathrm{n}=9-12) .{ }^{\#} p<0.05$ and ${ }^{\# \#} p<0.01$ compared with the $\mathrm{D}$-gal model group.

ming distance shortened progressively in five training days closely paralleled with the difference in escape latency: day, $F(4,164)$ $=55.288, p<0.01$; treatment, $\mathrm{F}(4,164)=42.247, p<0.01$; day by treatment interaction, $F(16,164)=1.324, p>0.05$. The D-gal model group had significantly longer swimming distance than the vehicle control group $(p<0.01)$ and all the treatment groups substantially shortened the swimming distance.

The platform crossing number and the time spent in the target quadrant after the 5 training days were recorded to evaluate the spatial learning and memory ability of mice on the 6th day, as shown in Fig. 4C-D. The platform crossing number for the D-gal model group was remarkably decreased as compared to those of the vehicle control $(p<0.01)$, and the time spent in the target quadrant was significantly less $(p<0.01)$. The impairment in the spatial learning and memory ability by D-gal were attenuated by the caffeic acid derivatives. Treatment with $10 \mathrm{mg} / \mathrm{kg} / \mathrm{d}$ CAPE, Danshensu or Curcumin for 2 weeks increased the number of crossing over the platform site and the time spent in the target quadrant $(p<0.05 ; p<0.01)$. There was no significant difference between the Curcumin and Danshensu group mice $(p<0.01)$. Taken together, treatment with all three caffeic acid derivatives could improve spatial learning and memory deficits in Dgal-treated mice.

3.4. Effects of three caffeic acid derivatives on MDA content and SOD, GPX and Catalase activities in hippocampus of senescent mice induced by $D$-gal

Table 1 summarized the effects of CAPE, Curcumin and Danshensu on MDA levels and SOD, Gpx, Catalase activities in hippocampus. D-gal model group showed significant decrease in the activities of SOD, Gpx and Catalase, and increased MDA levels as compared with the vehicle control group.

All the three caffeic acid derivatives exhibited significant effects on elevating the activities of SOD, Gpx and Catalase, as well as on reducing the MDA levels in mice hippocampi as compared with the D-gal model group $(p<0.05 ; \quad p<0.01 ; \quad p<0.001)$, which was CAPE $>$ Danshensu $>$ Curcumin. However, Curcumin showed no effect on the activities of SOD or increase in the levels of MDA in Curcumin group as compared with the D-gal model group.
(A)

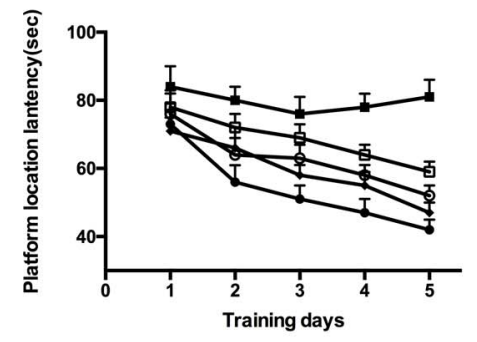

(C)

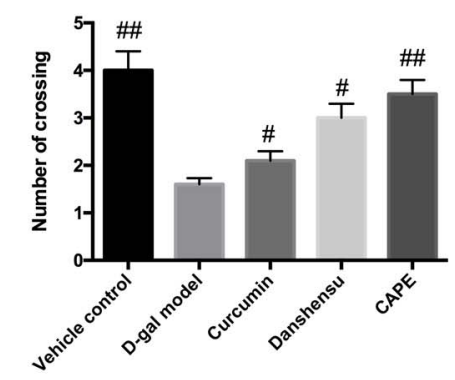

(B)

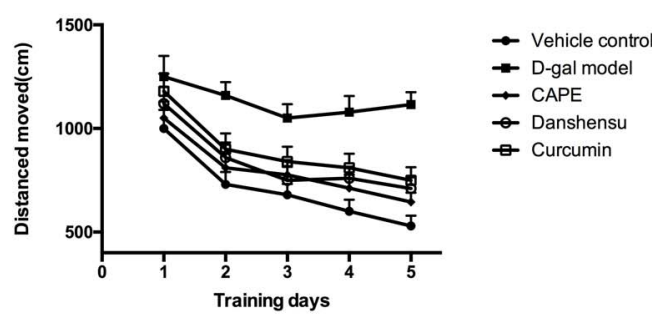

(D)

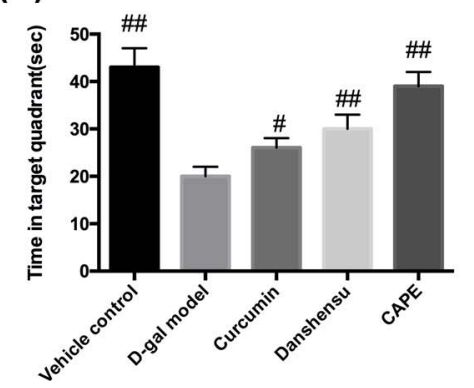

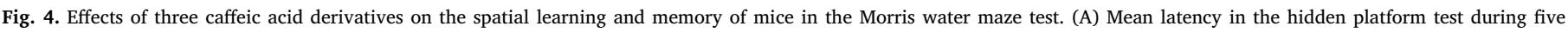

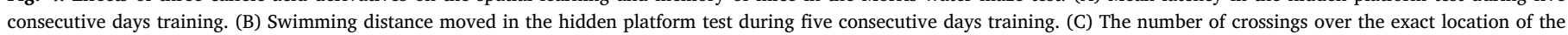
former platform. (D) Time spent in the target quadrant. All values are expressed as means \pm SEM (n $=6-7$ ). ${ }^{\#} p<0.05$ and ${ }^{\# \#} p<0.01$ compared with the D-gal model group. 
Table 1

Effect of three caffeic acid derivatives on MDA levels and activities of SOD, Gpx and Catalase in the hippocampus.

\begin{tabular}{|c|c|c|c|c|}
\hline Group & MDA (n M/mg protein) & SOD (U/mg protein) & Gpx (U/mg protein) & Catalase (U/g protein) \\
\hline Vehicle control & $5.43 \pm 0.34^{\# \# \#}$ & $87.28 \pm 5.67^{\# \# \#}$ & $982.68 \pm 72.86^{\# \#}$ & $103.62 \pm 8.56^{\# \# \#}$ \\
\hline D-gal model & $7.81 \pm 0.38$ & $46.82 \pm 3.79$ & $686.80 \pm 34.27$ & $45.22 \pm 4.12$ \\
\hline CAPE & $5.83 \pm 0.48^{\# \# \#}$ & $68.85 \pm 5.54^{\# \#}$ & $902.76 \pm 73.56^{\# \#}$ & $90.12 \pm 7.96^{\# \#}$ \\
\hline Danshensu & $6.23 \pm 0.25^{\#}$ & $62.43 \pm 4.09^{\#}$ & $801.27 \pm 63.75^{\# \#}$ & $70.78 \pm 6.24^{\# \#}$ \\
\hline Curcumin & $7.04 \pm 0.54$ & $54.56 \pm 4.25$ & $737.82 \pm 57.17^{\#}$ & $60.54 \pm 5.67^{\#}$ \\
\hline
\end{tabular}

All values are means \pm S.E.M. $(\mathrm{n}=6-7) .{ }^{\#} p<0.05 ;{ }^{\# \#} p<0.01 ;{ }^{\# \# \#} p<0.001$ compared with the $\mathrm{D}$-gal model group.

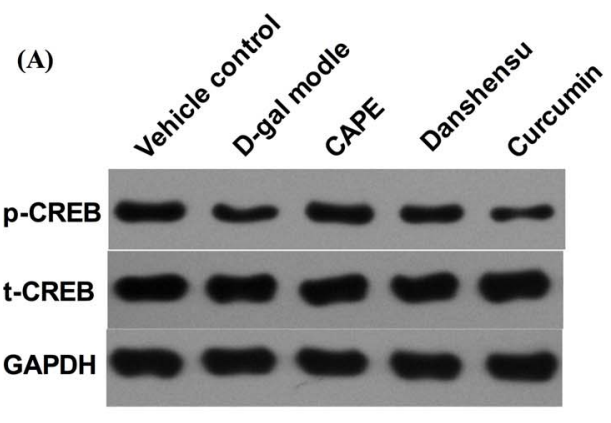

(C)

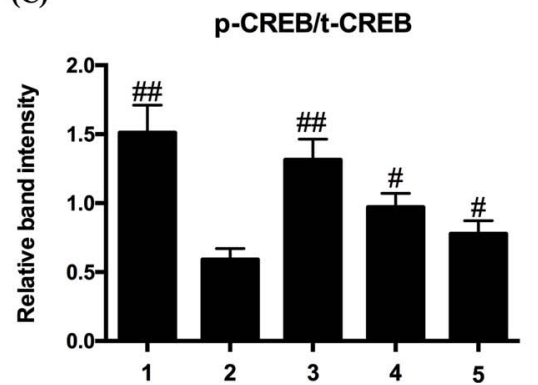

(B)
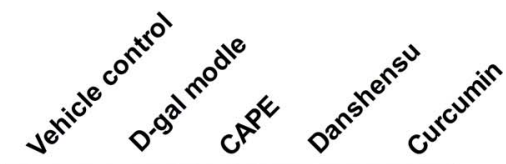

p-PKA

t-PKA

GAPDH

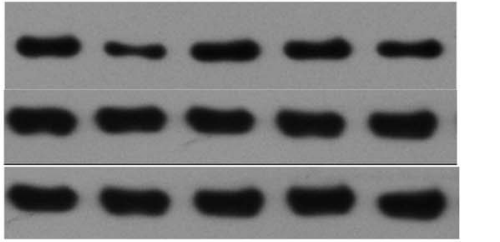

(D)

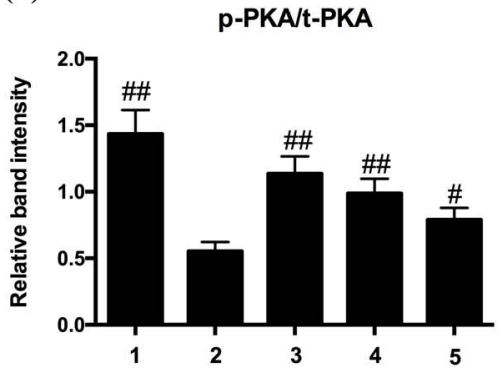

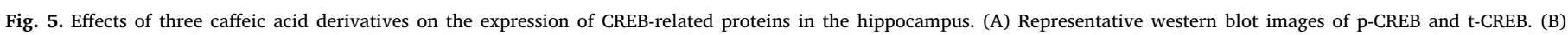

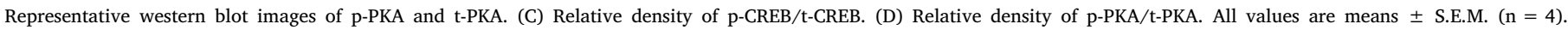
${ }^{\#} p<0.05 ;{ }^{\# \#} p<0.01$ compared with the D-gal model group. 1, Vehicle control group. 2, D-gal model group. 3, CAPE group. 4, Danshensu group. 5, Curcumin group.

\subsection{Western blotting}

As shown in Fig. 5, western blot analysis revealed that CREB phosphorylation in the D-gal model group was reduced as compared to the Vehicle control group. CAPE, Danshensu, and Curcumin significantly rescued the D-gal-induce impairment of CREB activation in hippocampi of mice treated with D-gal ( $p<0.05$; $p<0.01$ ), which was CAPE $>$ Danshensu $>$ Curcumin. We also examined the expression levels of phosphorylated PKA, the major kinase phosphorylating CREB at Ser133. The level of phospho-PKA was significantly lower in the D-gal model group compared with the vehicle control group $(p<0.01)$, whereas pretreatment with CAPE and Danshensu restored phospho-PKA levels to vehicle control group.

\section{Discussion}

Neurodegenerative diseases such as Alzheimer's disease, Parkinson's disease and other dementias are becoming a scourge due to the unprecedented increase in human life expectancy. Neurodegenerative diseases represent one of the main causes of death in the industrialized countries [21].

It is reported that ROS play a critical role in the pathogenesis of neurodegenerative disease $[22,23]$. ROS in response to the apoptotic process include activation of caspases, activation of nucleases and inactivation of nuclear repair polymerases. At physiological levels, ROS function as "redox messengers" in intracellular signaling, while excess
ROS induce oxidative modification of cellular macromolecules, inhibit protein function and promote cell death. In an effort to better understand the role of oxidative stress in disease progression, many studies have investigated the effects of natural products as antioxidants in vitro and in vivo. The present study reports for the protective effect of three caffeic acid derivatives against $\mathrm{H}_{2} \mathrm{O}_{2}$-induced oxidative damage in PC12 cells and D-gal induced neurotoxicity in mice.

We first investigated the neuroprotective activity of the caffeic acid derivatives against $\mathrm{H}_{2} \mathrm{O}_{2}$-induced PC12 cells injury by MTT assay. $\mathrm{H}_{2} \mathrm{O}_{2}$ has been previously shown to induce apoptosis in PC12 cells, and antioxidant treatments had protective effect against $\mathrm{H}_{2} \mathrm{O}_{2}$ induced cell injury $[24,25]$. Thus, antioxidant treatment may provide useful intervention strategies to reduce oxidative stress induced cell injury. In this study, PC12 cells exposed to $\mathrm{H}_{2} \mathrm{O}_{2}$ displayed significant reduction in cell viability. Pretreatment with caffeic acid derivatives exhibited potent protection against $\mathrm{H}_{2} \mathrm{O}_{2}$-induced cytotoxicity.

Since caffeic acid derivatives treatment exhibited distinct protective effect against $\mathrm{H}_{2} \mathrm{O}_{2}$-induced oxidative damage in PC12 cells, the study was further extended to in vivo conditions using a D-gal induced neurotoxicity mouse model.

Previous studies demonstrated that D-gal-treated mice showed agerelated deficits in a series of behavioral tests [26,27]. In this study, the effects of CAPE, Danshensu and Curcumin on the D-gal-induced neurotoxicity were first investigated by behavioral tests. Significant differences between the vehicle control mice and the D-gal model mice were observed in both Morris water maze test and step-down avoidance 
test, suggesting that the injection of D-gal caused motor abnormalities and impairments of novelty-induced exploratory behaviors. These data also indicated that CAPE, Danshensu and Curcumin could ameliorate the cognitive impairment induced by D-gal.

Lastly, in order to investigate the neuroprotective mechanism of the caffeic acid derivatives on D-gal-treated mice, we studied the antioxidant activity in mouse hippocampus, a part of the mammalian brain involved in learning and memory [28]. The human body has a complex antioxidant defense system which contains the antioxidant enzymes SOD, GHP-Px and catalase. Any factor that can damage the activities of antioxidant enzymes may lead to accumulation of ROS and subsequently cause oxidative damage [29]. Meanwhile, ROS production can be indirectly estimated by measuring MDA, a product of lipid peroxidation [30]. In the present study, lowered SOD, GPx, catalase and elevated MDA content in the hippocampus of the D-gal model mice were observed as compared with the vehicle control mice. CAPE, Danshensu and Curcumin significantly increased of these antioxidative enzymes and decreased the level of MDA in the hippocampi of D-gal-treated mice. These results strongly suggested that CAPE, Danshensu and Curcumin could enhance the capacity of the mouse brain to against free radicals induced by D-gal.

Furthermore, in order to assess molecular changes associated with improved cognitive functioning induced by the caffeic acid derivatives, we evaluated the phosphorylation status of CREB and PKA. CREB an important target of PKA and also critical for several cellular processes including proliferation and adaptive responses [31]. Recent studies have shown that activation of the PKA/CREB pathway is a necessary step for the learning and memory [32]. Our western blotting observations show that CAPE, Danshensu and Curcumin can reverse D-galinduced impairment of PKA and CREB activations in mice hippocampi, suggesting that these three natural caffeic acid derivatives were able to exert neuroprotective effects via increasing the phosphorylations of PKA and CREB.

Our results suggest that all of the three caffeic acid derivatives could protect $\mathrm{H}_{2} \mathrm{O}_{2}$-induced cytotoxicity in PC12 cells and reduce the level of oxidative stress in vivo. CAPE, Danshensu and Curcumin could activate the phosphorylation of PKA and CREB. It is interesting to note that, the presence of 3, 4-dihydroxyphenyl groups in the A ring (Danshensu and CAPE) seems to correlate with their effect of up-regulating the expression of PKA-CREB pathway. These findings suggest that 3, 4dihydroxyphenyl hydroxyl groups in A ring plays a more important role than conjugated double bond in up-regulating the hippocampus expression of PKA/CREB pathway in the mouse hippocampus.

\section{Conclusion}

In conclusion, the present study revealed that CAPE, Danshensu and Curcumin exhibited potent protective effect against $\mathrm{H}_{2} \mathrm{O}_{2}$-induced cytotoxicity in PC12 cells and D-gal induced neurotoxicity in mice hippocampi, which may be mediated by their antioxidant activities and activating the PKA/CREB signaling pathway. The presence of 3, 4dihydroxyls in A ring of caffeic acid derivatives is necessary for their neuroprotective activity, while the conjugated bond might not contribute to up-regulation of the hippocampus expression of PKA/CREB pathway in the D-gal-induced cognitive impairment mice model.

\section{Conflict of interest}

The authors declare that they do not have any conflicts of interest.

\section{Acknowledgments}

This work was supported by the following grants: National Science Foundation of China (30973864) and Natural science foundation of Hubei province (2015CFB647).

\section{References}

[1] B. Uttara, A.V. Singh, P. Zamboni, R.T. Mahajan, Oxidative stress and neurodegenerative diseases: a review of upstream and downstream antioxidant therapeutic options, Curr. Neuropharmacol. 7 (2009) 65-74.

[2] A. Castegna, M. Aksenov, M. Aksenova, V. Thongboonkerd, J.B. Klein, W.M. Pierce, R. Booze, W.R. Markesbery, D.A. Butterfield, Proteomic identification of oxidatively modified proteins in Alzheimer's disease brain. Part I: creatine kinase BB, glutamine synthase, and ubiquitin carboxy-terminal hydrolase L-1, Free Radic. Biol. Med. 33 (2002) 562-571.

[3] B. Halliwell, Role of free radicals in the neurodegenerative diseases: therapeutic implications for antioxidant treatment, Drugs Aging 18 (2001) 685-716.

[4] K. Jomova, M. Valko, Advances in metal-induced oxidative stress and human disease, Toxicology 10 (283) (2011) 65-687.

[5] E.R. Kandel, The molecular biology of memory: cAMP, PKA, CRE, CREB-1, CREB-2 and CPEB, Mol. Brain 5 (2012) 14-26.

[6] M.P. Delghandi, M. Johannessen, U. Moens, The cAMP signaling pathway activates CREB through PKA: p38 and MSK1 in NIH 3T3 cells, Cell. Signal. 17 (2005) 1343-1351.

[7] A.F. Teich, R.E. Nicholls, D. Puzzo, J. Fiorito, R. Purgatorio, M. Fa', O. Arancio, Synaptic therapy in Alzheimer's disease: a CREB-centric approach, Neurotherapeutics 12 (2015) $29-41$.

[8] Y. Chen, X. Huang, Y.W. Zhang, E. Rockenstein, G. Bu, T.E. Golde, E. Masliah, H. Xu, Alzheimer's $\beta$-secretase (BACE1) regulates the cAMP/PKA/CREB pathway independently of $\beta$-amyloid, J. Neurosci. 32 (2013) 11390-11395.

[9] T. Silva, C. Oliveira, F. Borges, Caffeic acid derivatives, analogs and applications: a patent review (2009-2013), Expert Opin. Ther. Pat. 24 (2014) 1257-1270.

[10] R.W. Jiang, K.M. Lau, P.M. Hon, T.C. Mak, K.S. Woo, K.P. Fung, Chemistry and biological activities of caffeic acid derivatives from Salvia miltiorrhiza, Curr. Med. Chem. 12 (2005) $237-246$.

[11] H. Parlakpinar, E. Sahna, A. Acet, B. Mizrak, A. Polat, Protective effect of caffeic acid phenethyl ester (CAPE) on myocardial ischemia-reperfusion-induced apoptotic cell death, Toxicology 209 (2005) 1-14.

[12] N. Kitsati, D. Fokas, M.D. Ouzouni, M.D. Mantzaris, A. Barbouti, D. Galaris, Lipophilic caffeic acid derivatives protect cells against $\mathrm{H} 2 \mathrm{O} 2$-Induced DNA damage by chelating intracellular labile iron, J. Agric. Food Chem. 60 (2012) 7873-7879.

[13] Q. Du, C. Hao, J. Gou, X. Li, K. Zou, X. He, Z. Li, Protective effects of p-nitro caffeic acid phenethyl ester on acute myocardial ischemia-reperfusion injury in rats, Exp. Ther. Med. 11 (2016) 1433-1440.

[14] O. Li, Y.N. He, X.W. Shi, L.Y. Kang, L.Y. Niu, X.G. Wang, W. Feng, Clerodens E-J, antibacterial caffeic acid derivatives from the aerial part of Clerodendranthus spicatus, Fitoterapia 114 (2016) 110-114.

[15] M.B. Hansen, S.E. Nielsen, K. Berg, Re-examination and further development of a precise and rapid dye method for measuring cell growth/cell killing, J. Immunol. Methods 119 (1989) 203-210.

[16] M.M. Bradford, A rapid and sensitive method for the quantitation of microgram quantities of protein utilizing the principle of protein-dye binding, Anal. Biochem. 72 (1976) 248-254.

[17] H. Ohkawa, N. Ohishi, K. Yagi, Assay for lipid peroxides in animal tissues by thiobarbituric acid reaction, Anal. Biochem. 95 (1979) 351-358.

[18] Y. Oyanagui, Reevaluation of assay methods and establishment of kit for superoxide dismutase activity, Anal. Biochem. 142 (1984) 290-296.

[19] D. Armstrong, R. Browne, The analysis of free radicals, lipid peroxides, antioxidant enzymes and compounds related to oxidative stress as applied to the clinical chemistry laboratory, Adv. Exp. Med. Biol. 366 (1994) 43-58.

[20] H. Aebi, Catalase in vitro, Methods Enzymol. 105 (1984) 121-126.

[21] D.R. Gustafson, M. Clare Morris, N. Scarmeas, R.C. Shah, J. Sijben, K. Yaffe, X. Zhu, New perspectives on Alzheimer's disease and nutrition, J. Alzheimers Dis. 46 (2015) $1111-1127$.

[22] V.I. Bunik, J.V. Schloss, J.T. Pinto, G.E. Gibson, A.J. Cooper, Enzyme-catalyzed side reactions with molecular oxygen may contribute to cell signaling and neurodegenerative diseases, Neurochem. Res. 32 (2007) 871-891.

[23] M. Enari, H. Sakahira, H. Yokoyama, K. Okawa, A. Iwamatsu, S. Nagata, A caspaseactivated DNase that degrades DNA during apoptosis and its inhibitor ICAD, Nature 391 (1998) 43-50.

[24] H.Y. Xue, D.Y. Niu, G.Z. Gao, Q.Y. Lin, L.J. Jin, Y.P. Xu, Aucubin modulates Bcl-2 family proteins expression and inhibits caspases cascade in $\mathrm{H}_{2} \mathrm{O}_{2}$-induced PC12 cells, Mol. Biol. Rep. 38 (2011) 3561-3567.

[25] Y. Fujita, Y. Izawa, N. Ali, Y. Kanematsu, K. Tsuchiya, S. Hamano, T. Tamaki, M. Yoshizumi, Pramipexole protects against $\mathrm{H}_{2} \mathrm{O}_{2}$-induced PC12 cell death, N-S Arch. Pharmacol. 372 (2006) 257-266.

[26] J. Tian, K. Ishibashi, K. Ishibashi, K. Reiser, R. Grebe, B.S. Shyam, P. Gehlbach, J.T. Handa, Advanced glycation endproduct-Induced aging of the retinal pigment epithelium and choroid: a comprehensive transcriptional response, Proc. Natl. Acad. Sci. U. S. A. 102 (2005) 11846-11851.

[27] K. Parameshwaran, M.H. Irwin, K. Steliou, C.A. Pinkert, D-Galactose effectiveness in modeling aging and therapeutic antioxidant treatment in mice, Rejuvenation Res. 13 (2010) 729-735.

[28] T.C. Foster, Biological markers of age-related memory deficits: treatment of senescent physiology, CNS Drugs 20 (2006) 153-166.

[29] K. Rahman, Studies on free radicals, antioxidants and co-factors, Clin. Interv. Aging 2 (2007) 219-236.

[30] K. Yagi, R. Rastogi, Assay for lipid peroxides in animal tissue by thiobarbituric acid reaction, Anal. Biochem. 95 (1979) 351-358.

[31] R. Waltereit, M. Weller, Signaling from cAMP/PKA to MAPK and synaptic plasticity, Mol. Neurobiol. 27 (2003) 99-106.

[32] B.E. Lonze, D.D. Ginty, Function and regulation of CREB family transcription factors in the nervous system, Neuron 35 (2002) 605-623.

[33] J. Rubio, H. Dang, M. Gong, X. Liu, S.L. Chen, G.F. Gonzales, Aqueous and hydroalcoholic extracts of Black Maca (Lepidium meyenii) improve scopolamine-induced memory impairment in mice, Food Chem. Toxicol. 45 (2007) 1882-1890. 